\title{
Experimental investigation of process parameters in WEDM of Al 7075 alloy
}

\author{
Kingshuk Mandal ${ }^{1, *}$, Dipankar Bose ${ }^{2}$, Souren Mitra ${ }^{1}$, Soumya Sarkar ${ }^{1}$ \\ ${ }^{1}$ Production Engineering Department, Jadavpur University, Kolkata 700032, India \\ ${ }^{2}$ Mechanical Engineering Department, NITTTR-Kolkata, Kolkata 700106, India
}

Received: 18 March 2020 / Accepted: 13 July 2020

\begin{abstract}
Process parameters selection is a critical task in wire electro-discharge machining (WEDM). The parameter settings would vary when a material to be machined is changed. In the present study, experimental investigation has been carried out for appropriate selection of process parameters in WEDM of $\mathrm{Al}$ 7075. It has been perceived that pulse on time $\left(\mathrm{T}_{\mathrm{on}}\right)$, arc on time $\left(\mathrm{A}_{\mathrm{on}}\right)$ pulse off time $\left(\mathrm{T}_{\mathrm{off}}\right)$, arc off time $\left(\mathrm{A}_{\text {off }}\right)$, servo sensitivity $\left(\mathrm{S}_{\mathrm{c}}\right)$, wire tension $\left(\mathrm{W}_{\mathrm{t}}\right)$ and servo voltage $\left(\mathrm{S}_{\mathrm{v}}\right)$ are the major influencing factors on machining speed $\left(V_{c}\right)$, corner error $\left(C_{e}\right)$ and surface roughness $\left(R_{a}\right)$ for this alloy. It is also observed that selected seven process parameters are essential for effective machining of Al 7075 alloy. Finally, three-dimensional (3D) surface topography has been analysed to determine the characteristics of the machined surface.
\end{abstract}

Keywords: Wire electro discharge machining / Al 7075 alloy / machining speed / corner error / surface roughness

\section{Introduction}

Al 7075, commonly known as a light-metal alloy, is a new generation metal alloy that has excellent mechanical properties and exhibits high strength, high toughness and good ductility, and it also renders good resistance to fatigue and corrosion. Al 7075 is widely used in aircraft engine cylinder, frames and intricate vehicle sprocket in automobile industries [1]. The dimensional accuracy including surface finish is extremely important aspects while machining of such beneficial materials. However, at the same time $\mathrm{Al} 7075$ is difficult to machine by conventional technique while maintaining the required precision, surface finish and dimensional tolerances. Wire electro-discharge machining (WEDM) is a potential solution for machining such high strength electrically conductive material. WEDM is the process where a moving electrode (wire) continuously transforms electrical energy into thermal energy in the form of visible spark for machining materials $[2,3]$. Due to this nature, any kind of electrically conductive materials, metal matrix composites (MMCs) and low conductive ceramics can be efficiently machined regardless to their mechanical or chemical properties $[4,5]$. Furthermore, WEDM is capable to produce any kind of complex shape with high precision

\footnotetext{
* e-mail: mandal.kingshuk1@gmail.com
}

and good quality of surface finish [6,7]. In this research work, influence of the input factors in wire electrodischarge machining (WEDM) of Al 7075 alloy has been reported. Machining speed, corner accuracy and surface finish are considered as performance characteristics. In fact, these response parameters determine the quality and productivity of the manufacturing components in WEDM.

A wide range of the past research works in the field of WEDM revealed the effect of input parameters on material removal rate (MRR) and the surface characteristics [8]. A few number of research works have been reported on wire lag phenomenon during rough cutting operation in WEDM, that is an important and deciding factor to explore the corner accuracy [9]. However, a very limited number of research works are available on the influence of process parameters and optimization on dimensional accuracy [10]. Nevertheless, this response parameter is extremely important for achieving high precision job in WEDM. A small number of research works have been reported in WEDM of $\mathrm{Al} 7075$ based MMCs [11,12]. Furthermore, the effect of arc on time $\left(\mathrm{A}_{\mathrm{on}}\right)$, servo sensitivity $\left(\mathrm{S}_{\mathrm{c}}\right)$ and arc off time $\left(\mathrm{A}_{\text {off }}\right)$ on machining performance, especially on corner error $\left(\mathrm{C}_{\mathrm{e}}\right)$ and other responses have not been reported so far in the field of WEDM for this alloy. The objective of the present research work is to carry out the experimental investigation of different input factors, namely pulse on time $\left(\mathrm{T}_{\mathrm{on}}\right)$, arc on time $\left(\mathrm{A}_{\text {on }}\right)$, pulse off time $\left(\mathrm{T}_{\text {off }}\right)$, arc off time $\left(\mathrm{A}_{\text {off }}\right)$, servo 


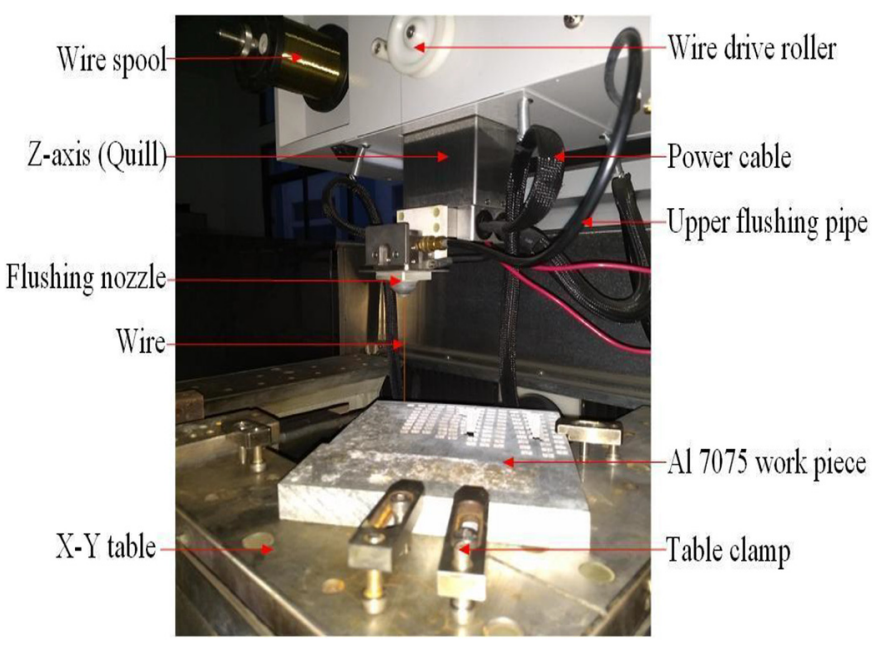

Fig. 1. Pictorial view of WEDM table unit (Model: EX 40).

sensitivity $\left(\mathrm{S}_{\mathrm{c}}\right)$, servo voltage $\left(\mathrm{S}_{\mathrm{v}}\right)$ and wire tension $\left(\mathrm{W}_{\mathrm{t}}\right)$ on machining speed $\left(\mathrm{V}_{\mathrm{c}}\right)$, corner error $\left(\mathrm{C}_{\mathrm{e}}\right)$ and surface roughness $\left(\mathrm{R}_{\mathrm{a}}\right)$ during single pass cutting operation.

\section{Experimentation}

\subsection{Experimental set-up and procedure}

The experimentations have been carried out in EX-40 WEDM system. Brass coated wire electrode with diameter of $250 \mu \mathrm{m}$ is used to perform the experiments. Deionized water has been used as dielectric and its temperature maintained at $21^{\circ} \mathrm{C}$. For maximum dielectric flow, both the upper and lower flushing nozzle are kept fully open. The pictorial view of WEDM table unit is shown in Figure 1.

The quality of the product manufactured by WEDM is affected by the machining parameters. Genichi Taguchi is developed a three-stage design methodology, to understand the importance of design of experiment [13]. In the present study, Taguchi based design technique has been used to perform the experiments. $\mathrm{L}_{18}$ orthogonal array (mixed) design technique have been chosen for the experiments. Here, three level design is used for six parameters and two level design for one parameter. The full factorial design for seven factors with three levels is L2187 $\left(3^{7}\right)$ orthogonal array (OA). However, in this case L18 OA fractional factorial design is used [14]. Based upon some preliminary investigation and past literature survey, pulse on time $\left(\mathrm{T}_{\mathrm{on}}\right.$ in $\left.\mu \mathrm{s}\right)$, arc on time $\left(\mathrm{A}_{\mathrm{on}}\right.$ in $\left.\mu \mathrm{s}\right)$, pulse off time ( $\mathrm{T}_{\text {off }}$ in $\left.\mu \mathrm{s}\right)$, arc off time $\left(\mathrm{A}_{\text {off }}\right.$ in $\left.\mu \mathrm{s}\right)$, servo sensitivity $\left(\mathrm{S}_{\mathrm{c}}\right)$, servo voltage $\left(\mathrm{S}_{\mathrm{v}}\right.$ in volt $)$ and wire tension $\left(\mathrm{W}_{\mathrm{t}}\right.$ in $\left.\mathrm{kg}\right)$ have been chosen as controllable factors. Nevertheless, in this case arc on time is found to be the least significant process parameter on corner error and surface roughness during trial run. At higher arc on time, frequently wire breakage and unstable machining are observed. For that reason, two level design of this factor is considered. The ranges and corresponding levels of input parameters are shown in Table 1 . The performance measures after the machining are assessed in terms of corner error $\left(\mathrm{C}_{e}\right.$ in $\left.\mu \mathrm{m}\right)$,
Table 1. Input factors and their levels.

\begin{tabular}{llllll}
\hline Parameters & Notations & Units & \multicolumn{3}{c}{ Levels } \\
\cline { 4 - 6 } & & & 1 & 2 & 3 \\
\hline Pulse on time & $\mathrm{T}_{\text {on }}$ & $\mu \mathrm{s}$ & 0.5 & 0.7 & 0.9 \\
Pulse off time & $\mathrm{T}_{\text {off }}$ & $\mu \mathrm{s}$ & 14 & 24 & 34 \\
Arc on time & $\mathrm{A}_{\text {on }}$ & $\mu \mathrm{s}$ & 0.2 & 0.3 & 0.4 \\
Arc off time & $\mathrm{A}_{\text {off }}$ & $\mu \mathrm{s}$ & 10 & 30 & - \\
Servo voltage & $\mathrm{S}_{\mathrm{v}}$ & $\mathrm{V}$ & 10 & 15 & 20 \\
Wire tension & $\mathrm{W}_{\mathrm{t}}$ & $\mathrm{kg}$ & 0.8 & 1.2 & 1.6 \\
Servo sensitivity & $\mathrm{S}_{\mathrm{c}}$ & - & 1 & 2 & 3 \\
\hline
\end{tabular}

surface roughness $\left(R_{a}\right.$ in $\left.\mu m\right)$ and machining speed $\left(\mathrm{V}_{\mathrm{c}}\right.$ in $\left.\mathrm{mm} / \mathrm{min}\right)$. The design of experiments (DOE) for respective input and output are given in Table 2 .

\subsection{Measurements}

Corner error $\left(\mathrm{C}_{\mathrm{e}}\right)$ of the machined surface along the cutting direction is measured by CV-3200 (Mitutoyo, Japan) high precision contour measuring instrument. Measurement of the corner profile has been taken along the bisector $\left(45^{\circ}\right)$ of the actual corner angle $\left(90^{\circ}\right)$. Schematic representation of the corner error measurement and the path produced by wire electrode are shown in Figure 2. The surface roughness value is measured by SJ-410 (Mitutoyo, Japan) contact type surface measuring instrument. 3D surface topography of the machined surface is captured by high-resolution CCI (Taylor \& Hobson, UK) microscope. Machining speed is observed from the monitor of the machine and average value is considered for analysis. Corner error and surface roughness measurement have been repeated five times to minimize the error and average value is taken. The required measurements have been taken in vibration free and stable temperature ambience to avoid the measurement error.

\section{Results and analysis}

\subsection{ANOVA for machining speed, corner error and surface roughness}

A statistical approach for estimation of the relative influence of each variable on overall measured response is analysis of variance (ANOVA). In general, the relative importance of individual factor is often represented by the term of $\mathrm{F}$-value [13]. The $\mathrm{F}$-value in analysis of variance plying most significant role for input factors. In this work, ANOVA has been employed to analysis the significance of seven input factors on machining speed, corner error and surface roughness. ANOVA results for $\mathrm{V}_{\mathrm{c}}, \mathrm{C}_{\mathrm{e}}$ and $\mathrm{R}_{\mathrm{a}}$ are given in Tables $3-5$.

From the ANOVA tables, it has been observed that pulse parameters (i.e. $\left.T_{\text {on }} \& T_{\text {off }}\right)$ and arc on time $\left(A_{\text {on }}\right)$ are the most dominating factors (i.e. larger the $\mathrm{F}$-value) on machining speed $\left(\mathrm{V}_{\mathrm{c}}\right)$, corner error $\left(\mathrm{C}_{\mathrm{e}}\right)$ and surface roughness $\left(\mathrm{R}_{\mathrm{a}}\right)$. Apart from the above parameters, servo sensitivity $\left(\mathrm{S}_{\mathrm{c}}\right)$ playing a vital role to determine the 
Table 2. $\mathrm{L}_{18}$ experimental plan matrix and results.

\begin{tabular}{|c|c|c|c|c|c|c|c|c|c|c|}
\hline \multirow[t]{2}{*}{ Sl. No. } & \multicolumn{7}{|c|}{ Input factors } & \multicolumn{3}{|c|}{ Responses } \\
\hline & $\mathrm{T}_{\text {on }}(\mu \mathrm{s})$ & $\mathrm{T}_{\text {off }}(\mu \mathrm{s})$ & $\mathrm{A}_{\text {on }}(\mu \mathrm{s})$ & $\mathrm{A}_{\text {off }}(\mu \mathrm{s})$ & $\mathrm{S}_{\mathrm{v}}(\mathrm{V})$ & $\mathrm{W}_{\mathrm{t}}(\mathrm{kg})$ & $\mathrm{S}_{\mathrm{c}}$ & $\mathrm{V}_{\mathrm{c}}(\mathrm{mm} / \mathrm{min})$ & $\mathrm{C}_{\mathrm{e}}(\mu \mathrm{m})$ & $\mathrm{R}_{\mathrm{a}}(\mu \mathrm{m})$ \\
\hline 1 & 0.5 & 14 & 0.2 & 10 & 10 & 0.8 & 1 & 1.25 & 81 & 2.998 \\
\hline 2 & 0.5 & 24 & 0.3 & 10 & 15 & 1.2 & 2 & 3.24 & 80 & 3.053 \\
\hline 3 & 0.5 & 34 & 0.4 & 10 & 20 & 1.6 & 3 & 2.78 & 51 & 3.001 \\
\hline 4 & 0.7 & 14 & 0.2 & 10 & 15 & 1.2 & 3 & 4.61 & 97 & 3.179 \\
\hline 5 & 0.7 & 24 & 0.3 & 10 & 20 & 1.6 & 1 & 0.93 & 68 & 3.125 \\
\hline 6 & 0.7 & 34 & 0.4 & 10 & 10 & 0.8 & 2 & 3.00 & 101 & 2.987 \\
\hline 7 & 0.9 & 14 & 0.3 & 10 & 10 & 1.6 & 2 & 3.80 & 69 & 3.242 \\
\hline 8 & 0.9 & 24 & 0.4 & 10 & 15 & 0.8 & 3 & 4.42 & 118 & 3.180 \\
\hline 9 & 0.9 & 34 & 0.2 & 10 & 20 & 1.2 & 1 & 0.91 & 62 & 3.217 \\
\hline 10 & 0.5 & 14 & 0.4 & 30 & 20 & 1.2 & 2 & 2.28 & 75 & 2.961 \\
\hline 11 & 0.5 & 24 & 0.2 & 30 & 10 & 1.6 & 3 & 3.19 & 85 & 2.953 \\
\hline 12 & 0.5 & 34 & 0.3 & 30 & 15 & 0.8 & 1 & 0.92 & 79 & 2.882 \\
\hline 13 & 0.7 & 14 & 0.3 & 30 & 20 & 0.8 & 3 & 3.53 & 114 & 3.022 \\
\hline 14 & 0.7 & 24 & 0.4 & 30 & 10 & 1.2 & 1 & 1.22 & 70 & 3.005 \\
\hline 15 & 0.7 & 34 & 0.2 & 30 & 15 & 1.6 & 2 & 2.32 & 63 & 2.987 \\
\hline 16 & 0.9 & 14 & 0.4 & 30 & 15 & 1.6 & 1 & 1.14 & 58 & 3.191 \\
\hline 17 & 0.9 & 24 & 0.2 & 30 & 20 & 0.8 & 2 & 2.32 & 95 & 3.188 \\
\hline 18 & 0.9 & 34 & 0.3 & 30 & 10 & 1.2 & 3 & 4.31 & 91 & 3.084 \\
\hline
\end{tabular}

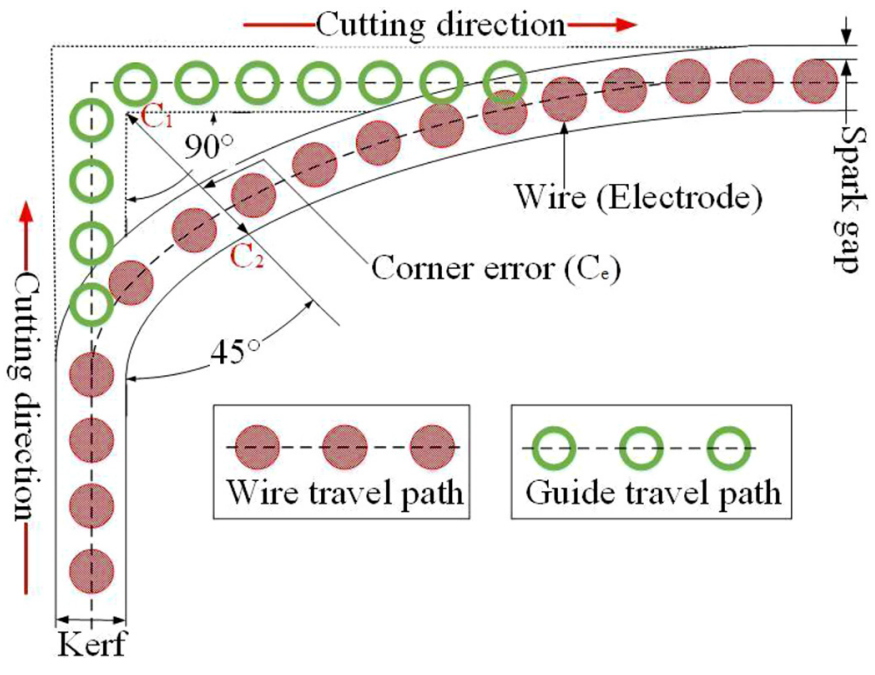

Fig. 2. Schematic representation of corner profile.

machining speed $(\mathrm{F}$-value $=120.23)$ and wire tension $\left(\mathrm{W}_{\mathrm{t}}\right)$ is playing a significant role on corner error $(\mathrm{F}$-value $=$ 114.21). Servo voltage $\left(\mathrm{S}_{\mathrm{v}}\right)$ is another important factor that have a moderate influence on machining speed $(\mathrm{F}$-value $=15.09), \quad$ surface roughness $\quad(\mathrm{F}$-value $=15.34)$ and high impact on corner error $(\mathrm{F}$-value $=62.05)$. In this work, analysis has been carried out in $95 \%$ confidence level.

\subsection{Modelling of the process parameters}

In this study, relations between input factors and output responses have been developed using regression technique.
In regression technique, the mathematical form of correlation between output and input are as follows:

$$
\mathrm{y}=\mathrm{f}\left(\mathrm{T}_{\mathrm{on}}, \mathrm{T}_{\text {off }}, \mathrm{A}_{\text {on }}, \mathrm{A}_{\text {off }}, \mathrm{S}_{\mathrm{v}}, \mathrm{W}_{\mathrm{t}}, \mathrm{S}_{\mathrm{c}}\right)
$$

where y defined, the output and f represent the output function. In the process of analysis, estimate of y has been calculated by using quadratic equation.

Mathematical model for corner error $\left(\mathrm{C}_{\mathrm{e}}\right)$, surface roughness $\left(\mathrm{R}_{\mathrm{a}}\right)$ and machining speed $\left(\mathrm{V}_{\mathrm{c}}\right)$ are the function of WEDM input parameters, in terms of pulse on time $\left(\mathrm{T}_{\text {on }}\right)$, arc on time $\left(\mathrm{A}_{\mathrm{on}}\right)$, pulse off time $\left(\mathrm{T}_{\mathrm{off}}\right)$, arc off time $\left(A_{\text {off }}\right)$, servo sensitivity $\left(S_{c}\right)$, servo voltage $\left(S_{v}\right)$ and wire tension $\left(\mathrm{W}_{\mathrm{t}}\right)$. The relation between inputs and outputs are established based on the experimental results. Insignificant process parameters $(\mathrm{P}$-value $>0.05)$ are eliminated from the model for better accuracy. The mathematical models for $\mathrm{V}_{\mathrm{c}}, \mathrm{C}_{\mathrm{e}}$ and $\mathrm{R}_{\mathrm{a}}$ are shown in Equation (2)-(4). The influence of the process parameters on output have been evaluated based on these mathematical models.

$$
\begin{aligned}
\mathrm{V}_{\mathrm{c}}= & -8.71+3.28 \mathrm{~T}_{\text {on }}-0.0281 \mathrm{~T}_{\text {off }}+20.30 \mathrm{~A}_{\text {on }} \\
& -0.02061 \mathrm{~A}_{\text {off }}+0.3110 \mathrm{~S}_{\mathrm{v}}+2.943 \mathrm{~S}_{\mathrm{c}}-1.38 \mathrm{~T}_{\text {on }}^{2} \\
& +0.000175 \mathrm{~T}_{\text {off }}^{2}-33.50 \mathrm{~A}_{\text {on }}^{2}-0.01260 \mathrm{~S}_{\mathrm{v}}^{2} \\
& -0.3925 \mathrm{~S}_{\mathrm{c}}^{2}
\end{aligned}
$$

$$
\begin{aligned}
\mathrm{C}_{\mathrm{e}}= & 50.3+150.8 \mathrm{~T}_{\text {on }}+1.35 \mathrm{~T}_{\text {off }}+66 \mathrm{~A}_{\text {on }}+0.57 \mathrm{~S}_{\mathrm{v}} \\
& -77.9 \mathrm{~W}_{\mathrm{t}}+10.67 \mathrm{~S}_{\mathrm{c}}-102.1 \mathrm{~T}_{\text {on }}^{2}-0.0333 \mathrm{~T}_{\text {off }}^{2} \\
& -108 \mathrm{~A}_{\text {on }}^{2}-0.0333 \mathrm{~S}_{\mathrm{v}}^{2}+16.7 \mathrm{~W}_{\mathrm{t}}^{2}+0.67 \mathrm{~S}_{\mathrm{c}}^{2}
\end{aligned}
$$


Table 3. ANOVA for cutting speed.

\begin{tabular}{|c|c|c|c|c|c|}
\hline Source & $\mathrm{DOF}$ & Adj SS & Adj MS & F-value & P-value \\
\hline Regression & 13 & 28.0241 & 2.15570 & 122.23 & 0.000 \\
\hline $\mathrm{T}_{\text {on }}$ & 1 & 0.848 & 0.8479 & 61.97 & 0.000 \\
\hline $\mathrm{T}_{\text {off }}$ & 1 & 0.436 & 0.4356 & 47.77 & 0.000 \\
\hline$A_{\text {on }}$ & 1 & 0.4537 & 0.45368 & 25.72 & 0.007 \\
\hline$A_{\text {off }}$ & 1 & 0.7647 & 0.76467 & 43.36 & 0.013 \\
\hline $\mathrm{S}_{\mathrm{v}}$ & 1 & 0.2662 & 0.26620 & 15.09 & 0.018 \\
\hline $\mathrm{W}_{\mathrm{t}}$ & 1 & 0.1046 & 0.10459 & 2.27 & $0.087^{*}$ \\
\hline $\mathrm{S}_{\mathrm{c}}$ & 1 & 2.1204 & 2.12040 & 120.23 & 0.000 \\
\hline $\mathrm{T}_{\mathrm{on}}^{2}$ & 1 & 0.4212 & 0.42120 & 30.69 & 0.002 \\
\hline $\mathrm{T}_{\text {off }}^{2}$ & 1 & 0.3211 & 0.32110 & 23.07 & 0.005 \\
\hline $\mathrm{A}_{\mathrm{on}}^{2}$ & 1 & 0.4489 & 0.44890 & 25.45 & 0.007 \\
\hline $\mathrm{S}_{\mathrm{v}}^{2}$ & 1 & 0.3969 & 0.39690 & 22.50 & 0.009 \\
\hline $\mathrm{W}_{\mathrm{t}}^{2}$ & 1 & 0.0481 & 0.04810 & 1.04 & $0.223^{*}$ \\
\hline $\mathrm{S}_{\mathrm{c}}^{2}$ & 1 & 0.6162 & 0.61623 & 34.94 & 0.004 \\
\hline Error & 4 & 0.0705 & 0.1764 & & \\
\hline Total & 17 & 28.0947 & & & \\
\hline \multicolumn{6}{|c|}{ Model summary } \\
\hline $\mathrm{S}$ & $\mathrm{R}^{2}$ & $\mathrm{R}^{2}(\operatorname{adj})$ & $\mathrm{R}^{2}$ (pred) & & \\
\hline 0.132801 & $99.75 \%$ & $98.93 \%$ & $92.82 \%$ & & \\
\hline
\end{tabular}

*Insignificant factors ( $\mathrm{P}$-value $>0.05)$.

Table 4. ANOVA for corner error.

\begin{tabular}{llllll}
\hline Source & DOF & Adj SS & Adj MS & F-value & P-value \\
\hline Regression & 13 & 5204.50 & 400.346 & 20.41 & 0.000 \\
$\mathrm{~T}_{\text {on }}$ & 1 & 173.79 & 173.786 & 134.76 & 0.000 \\
$\mathrm{~T}_{\text {off }}$ & 1 & 131.19 & 131.189 & 71.36 & 0.000 \\
$\mathrm{~A}_{\text {on }}$ & 1 & 144.77 & 144.771 & 106.24 & 0.000 \\
$\mathrm{~A}_{\text {off }}$ & 1 & 1.50 & 1.500 & 1.05 & $0.125^{*}$ \\
$\mathrm{~S}_{\mathrm{v}}$ & 1 & 73.88 & 73.884 & 62.05 & 0.012 \\
$\mathrm{~W}_{\mathrm{t}}$ & 1 & 214.94 & 214.939 & 114.21 & 0.000 \\
$\mathrm{~S}_{\mathrm{c}}$ & 1 & 27.86 & 27.864 & 14.12 & 0.032 \\
$\mathrm{~T}_{\text {on }}^{2}$ & 1 & 102.69 & 102.694 & 78.17 & 0.000 \\
$\mathrm{~T}_{\text {off }}^{2}$ & 1 & 44.44 & 44.444 & 20.27 & 0.008 \\
$\mathrm{~A}_{\text {on }}^{2}$ & 1 & 82.69 & 82.694 & 64.14 & 0.000 \\
$\mathrm{~S}_{\mathrm{v}}^{2}$ & 1 & 11.21 & 11.212 & 16.89 & 0.032 \\
$\mathrm{~W}_{\mathrm{t}}^{2}$ & 1 & 76.12 & 76.124 & 47.84 & 0.000 \\
$\mathrm{~S}_{\mathrm{c}}^{2}$ & 1 & 10.21 & 10.219 & 7.15 & 0.048 \\
Error & 4 & 78.44 & 19.611 & & \\
Total & 17 & 5282.94 & & & \\
Model summary & & & & \\
$\mathrm{S}$ & $\mathrm{R}^{2}$ & $\mathrm{R}^{2}($ adj $)$ & $\mathrm{R}^{2}($ pred $)$ & & \\
0.151044 & $98.52 \%$ & $94.69 \%$ & $89.93 \%$ & & \\
\hline
\end{tabular}

*Insignificant factors $(\mathrm{P}$-value $>0.05)$.
Table 5. ANOVA for surface roughness.

\begin{tabular}{llllll}
\hline Source & DOF & Adj SS & Adj MS & F-value & P-value \\
\hline Regression & 13 & 0.195271 & 0.015021 & 19.08 & 0.000 \\
$\mathrm{~T}_{\text {on }}$ & 1 & 0.072601 & 0.072601 & 76.21 & 0.000 \\
$\mathrm{~T}_{\text {off }}$ & 1 & 0.065727 & 0.065727 & 51.39 & 0.000 \\
$\mathrm{~A}_{\text {on }}$ & 1 & 0.050061 & 0.050061 & 34.75 & 0.005 \\
$\mathrm{~A}_{\text {off }}$ & 1 & 0.001128 & 0.001128 & 1.43 & $0.098^{*}$ \\
$\mathrm{~S}_{\mathrm{v}}$ & 1 & 0.021719 & 0.021719 & 15.34 & 0.018 \\
$\mathrm{~W}_{\mathrm{t}}$ & 1 & 0.007361 & 0.007361 & 4.14 & 0.038 \\
$\mathrm{~S}_{\mathrm{c}}$ & 1 & 0.030209 & 0.030209 & 11.58 & 0.021 \\
$\mathrm{~T}_{\text {on }}^{2}$ & 1 & 0.041601 & 0.041601 & 32.11 & 0.011 \\
$\mathrm{~T}_{\text {off }}^{2}$ & 1 & 0.033448 & 0.033448 & 23.17 & 0.034 \\
$\mathrm{~A}_{\text {on }}^{2}$ & 1 & 0.026200 & 0.026200 & 3.15 & 0.042 \\
$\mathrm{~S}_{\mathrm{v}}^{2}$ & 1 & 0.012687 & 0.012687 & 6.98 & 0.011 \\
$\mathrm{~W}_{\mathrm{t}}^{2}$ & 1 & 0.003200 & 0.003200 & 0.98 & $0.247^{*}$ \\
$\mathrm{~S}_{\mathrm{c}}^{2}$ & 1 & 0.000694 & 0.000694 & 0.12 & $0.085^{*}$ \\
Error & 4 & & & & \\
Total & 17 & & & & \\
Model summary & & & & \\
$\mathrm{S}$ & $\mathrm{R}^{2}$ & $\mathrm{R}^{2}($ adj) & $\mathrm{R}^{2}(\mathrm{pred})$ & & \\
0.028054 & $98.41 \%$ & $92.26 \%$ & $86.87 \%$ & & \\
\hline
\end{tabular}

*Insignificant factors ( $\mathrm{P}$-value $>0.05)$.

$$
\begin{aligned}
\mathrm{R}_{\mathrm{a}}= & 2.759-0.473 \mathrm{~T}_{\text {on }}+0.00665 \mathrm{~T}_{\text {off }}-0.326 \mathrm{~A}_{\text {on }} \\
& +0.0202 \mathrm{~S}_{\mathrm{v}}+0.354 \mathrm{~W}_{\mathrm{t}}-0.0012 \mathrm{~S}_{\mathrm{c}}+0.711 \mathrm{~T}_{\text {on }}^{2} \\
& -0.000214 \mathrm{~T}_{\text {off }}^{2}+0.27 \mathrm{~A}_{\text {on }}^{2}-0.000539 \mathrm{~S}_{\mathrm{v}}^{2}
\end{aligned}
$$

To verify the developed mathematical model, another set of confirmatory experiments have been carried out and results are given in Table 6 . Percentage of prediction error shown in the Table 7 is defined as follows:

$$
\begin{aligned}
& \% \text { prediction error } \\
& =\left\{\left\|\frac{\text { Experimental value }- \text { Predicted value }}{\text { Experimental value }}\right\|\right\} \times 100
\end{aligned}
$$

It has been perceived that the predicted results are closed to the experimental values. This proves the fact that the devolved mathematical models for machining of $\mathrm{Al}$ 7075 are suitable and effectively used for parametric analysis and optimization.

\subsection{Parametric study on machining speed, corner error and surface roughness}

3.3.1 Pulse on time $\left(T_{\text {on }}\right)$ and arc on time $\left(A_{\text {on }}\right)$

From Figure $3 \mathrm{a}$ and $3 \mathrm{~b}$, it is observed that the corner error $\left(\mathrm{C}_{\mathrm{e}}\right)$ machining speed $\left(\mathrm{V}_{\mathrm{c}}\right)$, and surface roughness $\left(\mathrm{R}_{\mathrm{a}}\right)$ all are increasing significantly with increase in pulse on time 
$\left(\mathrm{T}_{\text {on }}\right)$ and arc on time $\left(\mathrm{A}_{\mathrm{on}}\right)$. With increase of pulse on time and arc on time, energy per pulse increases and as a result, more material is removed per pulse. This discharge energy directly decided by pulse parameter settings (i.e. pulse on time and arc on time). These results in increased machining speed $\left(\mathrm{V}_{\mathrm{c}}\right)$ and also increase the gap force (force induced due to spark). At higher pulse on and arc on time, sparking occurred frequently and due to this frequent sparking, rate of melting material increases. This molten metal partially

Table 6. Verification experiments for mathematical model.

\begin{tabular}{|c|c|c|c|c|c|c|c|c|c|}
\hline \multicolumn{7}{|c|}{ Control factors } & \multicolumn{3}{|c|}{ Responses } \\
\hline $\mathrm{T}_{\text {on }}$ & $\mathrm{T}_{\text {off }}$ & $\mathrm{A}_{\text {on }}$ & $\mathrm{A}_{\text {off }}$ & $\mathrm{S}_{\mathrm{v}}$ & $\mathrm{W}_{\mathrm{t}}$ & $\mathrm{S}_{\mathrm{c}}$ & $\mathrm{V}_{\mathrm{c}}$ & $\mathrm{C}_{\mathrm{e}}$ & $\mathrm{R}_{\mathrm{a}}$ \\
\hline 0.5 & 34 & 0.4 & 30 & 20 & 1.6 & 3 & 2.39 & 71 & 2.905 \\
\hline 0.7 & 24 & 0.3 & 30 & 15 & 1.2 & 2 & 3.28 & 85 & 3.045 \\
\hline 0.9 & 14 & 0.2 & 10 & 10 & 0.8 & 1 & 1.83 & 87 & 3.217 \\
\hline 0.5 & 14 & 0.4 & 10 & 20 & 0.8 & 2 & 2.31 & 83 & 2.917 \\
\hline 0.9 & 34 & 0.2 & 30 & 15 & 1.6 & 2 & 2.68 & 64 & 3.012 \\
\hline
\end{tabular}

flushed out due to the high-pressure dielectric flushing and remaining materials stacked on the work surface. As a result lumping of molten metal takes place which forms different types of globule and making bigger crater in the machining zone. For that reason, the corner error $\left(\mathrm{C}_{e}\right)$ and surface roughness $\left(R_{a}\right)$ both are increasing with the increase of pulse on time $\left(\mathrm{T}_{\mathrm{on}}\right)$ and $\operatorname{arc}$ on time $\left(\mathrm{A}_{\mathrm{on}}\right)$.

\subsubsection{Pulse off time $\left(T_{\text {off }}\right)$ and arc off time $\left(A_{\text {off }}\right)$}

At the same time from Figure $4 \mathrm{a}$ and $4 \mathrm{~b}$, it is seen that corner error $\left(\mathrm{C}_{\mathrm{e}}\right)$, machining speed $\left(\mathrm{V}_{\mathrm{c}}\right)$, and surface roughness $\left(R_{a}\right)$ all are decreasing with increase in arc off time $\left(\mathrm{A}_{\text {off }}\right)$ and pulse off time $\left(\mathrm{T}_{\text {off }}\right)$. Increase in pulse off and arc off time results in less aggressive pulse parameter settings and as such, these results in decreased machining speed $\left(V_{c}\right)$ and surface roughness $\left(R_{a}\right)$. Due to less aggressive pulse parameters setting, the gap force as well as wire deflection reduces, which in turn helps in reducing the corner error $\left(\mathrm{C}_{\mathrm{e}}\right)$. Similarly, smaller sizes craters are induced within the machining zone; as a result, surface roughness $\left(R_{a}\right)$ decreases. It is good strategy of machining at higher pulse off parameter (i.e. $T_{\text {off }} \& A_{\text {off }}$ ) setting for better corner accuracy and surface finish, but it will cause

Table 7. Comparison between experimental results and model prediction values.

\begin{tabular}{|c|c|c|c|c|c|c|c|c|}
\hline \multicolumn{3}{|c|}{ Cutting speed $\left(\mathrm{V}_{\mathrm{c}}\right)$} & \multicolumn{3}{|c|}{ Corner error $\left(\mathrm{C}_{\mathrm{e}}\right)$} & \multicolumn{3}{|c|}{ Surface roughness $\left(R_{a}\right)$} \\
\hline $\begin{array}{l}\text { Experimental } \\
\text { result }\end{array}$ & $\begin{array}{l}\text { Predicted } \\
\text { value }\end{array}$ & $\%$ error & $\begin{array}{l}\text { Experimental } \\
\text { result }\end{array}$ & $\begin{array}{l}\text { Predicted } \\
\text { value }\end{array}$ & $\%$ error & $\begin{array}{l}\text { Experimental } \\
\text { result }\end{array}$ & $\begin{array}{l}\text { Predicted } \\
\text { value }\end{array}$ & $\%$ error \\
\hline 2.39 & 2.43 & 1.67 & 71 & 73 & 2.82 & 2.905 & 2.924 & 0.65 \\
\hline 3.28 & 3.19 & 2.74 & 85 & 88 & 3.53 & 3.045 & 3.098 & 1.74 \\
\hline 1.83 & 1.89 & 3.28 & 87 & 84 & 3.45 & 3.217 & 3.258 & 1.27 \\
\hline 2.31 & 2.42 & 4.76 & 79 & 82 & 3.79 & 2.917 & 3.016 & 3.41 \\
\hline 2.68 & 2.55 & 4.45 & 64 & 61 & 4.68 & 3.012 & 3.145 & 4.42 \\
\hline
\end{tabular}

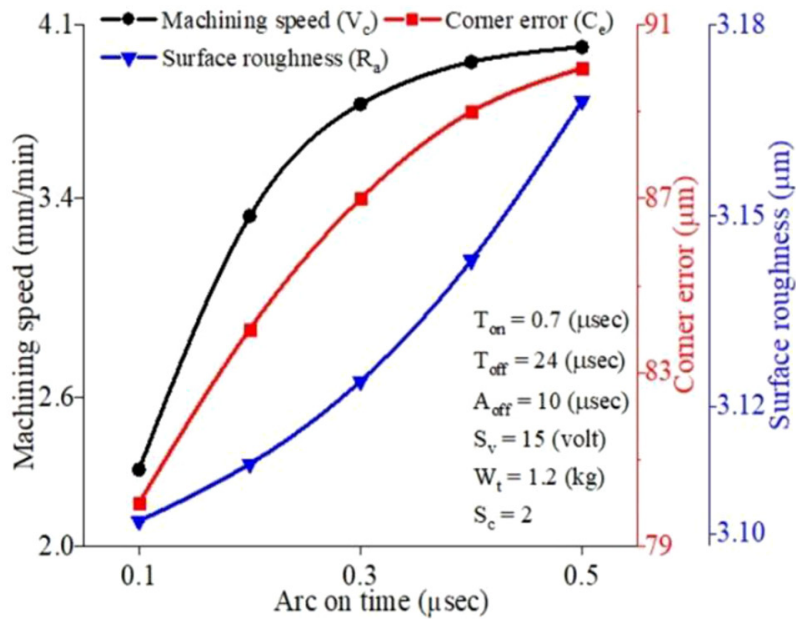

(a)

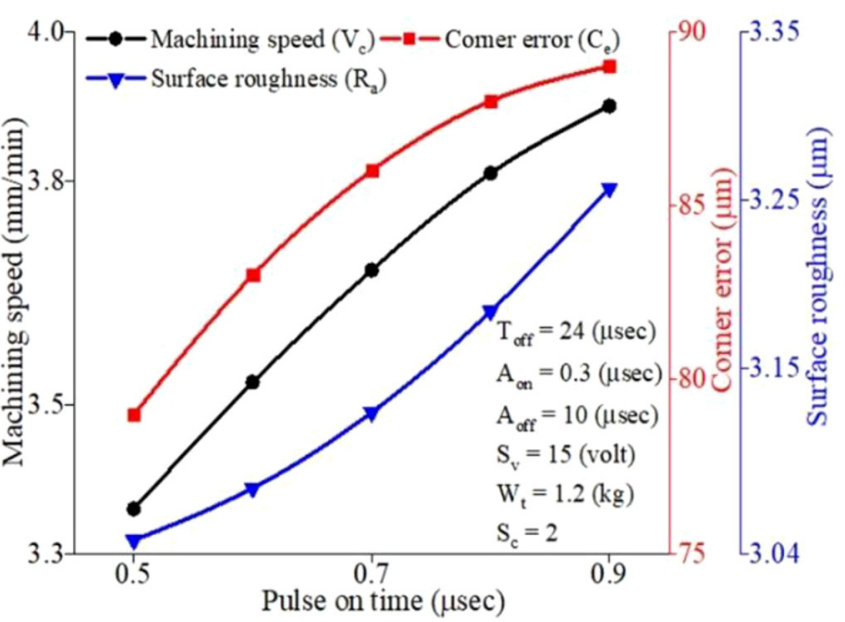

(b)

Fig. 3. Effect of (a) arc on time (b) pulse on time and on machining speed, corner error and surface roughness. 


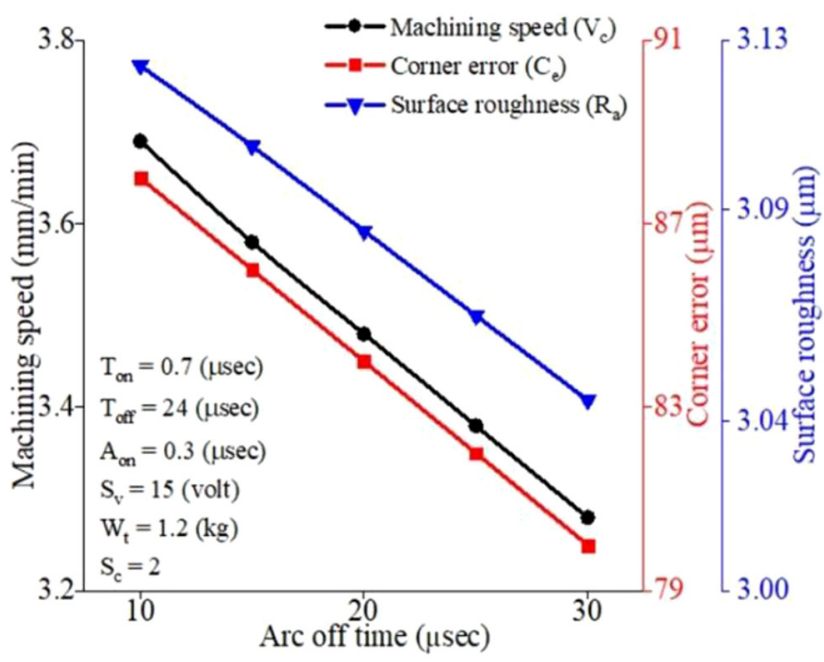

(a)

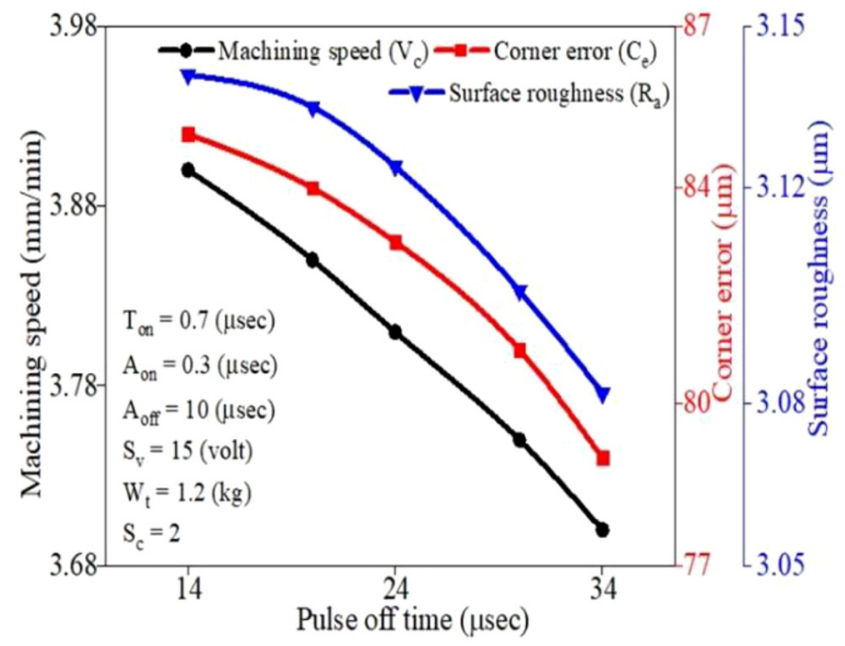

(b)

Fig. 4. Effect of (a) arc off time (b) pulse off time and on machining speed, corner error and surface roughness.

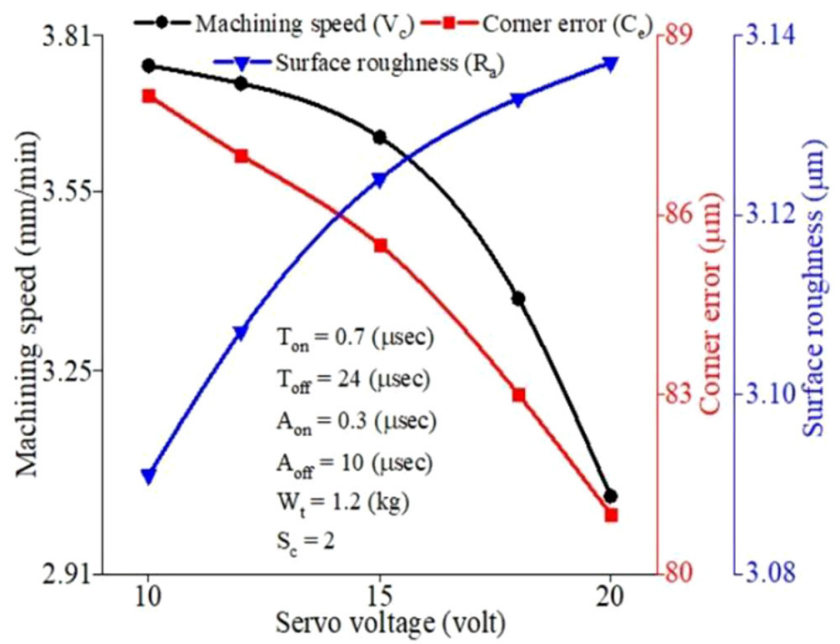

(a)

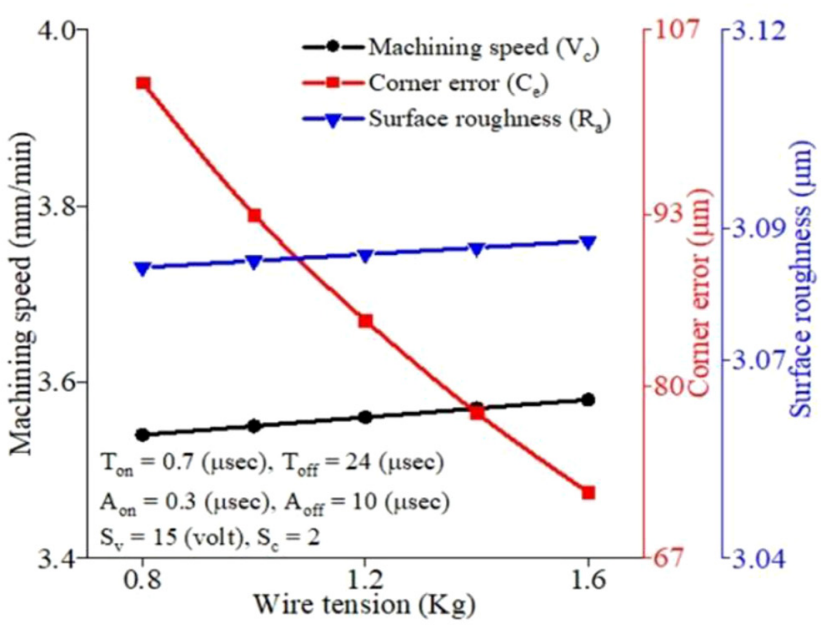

(b)

Fig. 5. Effect of (a) servo voltage and (b) wire tension on machining speed, corner error and surface roughness.

frequently wire breakage and unstable machining. The maximum possible upper limits of pulse off time and arc off time for this material have been set to avoid these kinds of problems.

\subsubsection{Servo voltage $\left(\mathrm{S}_{\mathrm{v}}\right)$}

It is observed from Figure 5 a that with increase in servo voltage $\left(\mathrm{S}_{\mathrm{v}}\right)$ both machining speed $\left(\mathrm{V}_{\mathrm{c}}\right)$ and corner error $\left(\mathrm{C}_{\mathrm{e}}\right)$ decreases but surface roughness $\left(\mathrm{R}_{\mathrm{a}}\right)$ increases. This can be explained by the fact that, with increasing servo voltage (i.e. desired gap voltage) the inter electrode gap voltage also increases, which in turn increases the gap between two electrodes. Due to this increased inter electrode gap, the proportion of pulse energy absorbed by the dielectric also increases. This results in less efficient utilization of pulse energy and consequently less machining speed. On the other hand, during sparking the gap force generated between two electrodes are transferred to the workpiece, wire and dielectric. With increasing inter electrode gap, amount of force transferred to dielectric tends to be more compared to that of wire. Due to this phenomenon, there will be less wire deflection and consequently less corner error $\left(\mathrm{C}_{\mathrm{e}}\right)$ due to increased servo voltage $\left(\mathrm{S}_{\mathrm{v}}\right)$. As already mentioned that increased servo voltage $\left(S_{v}\right)$ results in increased gap voltage, which in turns promote spark discharge at higher voltage, which contains more energy. These again produce bigger crater and consequently higher surface roughness $\left(R_{a}\right)$ value.

\subsubsection{Wire tension $\left(\mathrm{W}_{\mathrm{t}}\right)$}

Figure 5b it is observed that with increase in wire tension $\left(\mathrm{W}_{\mathrm{t}}\right)$ corner error $\left(\mathrm{C}_{\mathrm{e}}\right)$ reduces significantly, the wire deflection due to gap force reduces and as such, corner error reduces. However, no such significant effect has been observed 
on machining speed $\left(\mathrm{V}_{\mathrm{c}}\right)$ and surface roughness $\left(\mathrm{R}_{\mathrm{a}}\right)$ during machining of $\mathrm{Al} 7075$ alloy. Nevertheless, it is required to give a limiting value of wire tension to avoid the wire breakage phenomenon under stable machining condition.

\subsubsection{Servo sensitivity $\left(S_{c}\right)$}

It is observed from Figure 6 that with increase in servo sensitivity $\left(\mathrm{S}_{\mathrm{c}}\right)$ both machining speed $\left(\mathrm{V}_{\mathrm{c}}\right)$ and corner error $\left(\mathrm{C}_{\mathrm{e}}\right)$ increases but surface roughness $\left(\mathrm{R}_{\mathrm{a}}\right)$ decreases. The servo sensitivity is the intensity with which the WEDM controller reacts (in respect of the machining speed) in response to the change in error voltage between the desired gap voltage (servo voltage) and actual voltage. Thus, with increase in servo sensitivity $\left(\mathrm{S}_{\mathrm{c}}\right)$ for same error voltage (i.e. for same parameters setting) machining speed $\left(\mathrm{V}_{\mathrm{c}}\right)$ tends to be more to correct the error in gap voltage which in turn reduces the gap voltage and consequently the interelectrode gap (IEG). Thus, the spark discharge take place at comparatively lower voltage that will contain

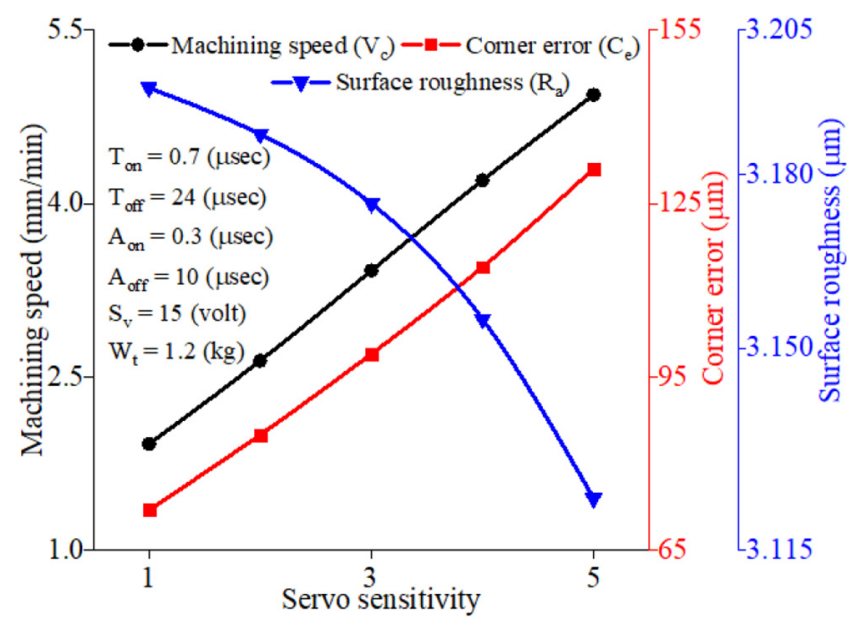

Fig. 6. Effect of servo sensitivity on machining speed, corner error and surface roughness.

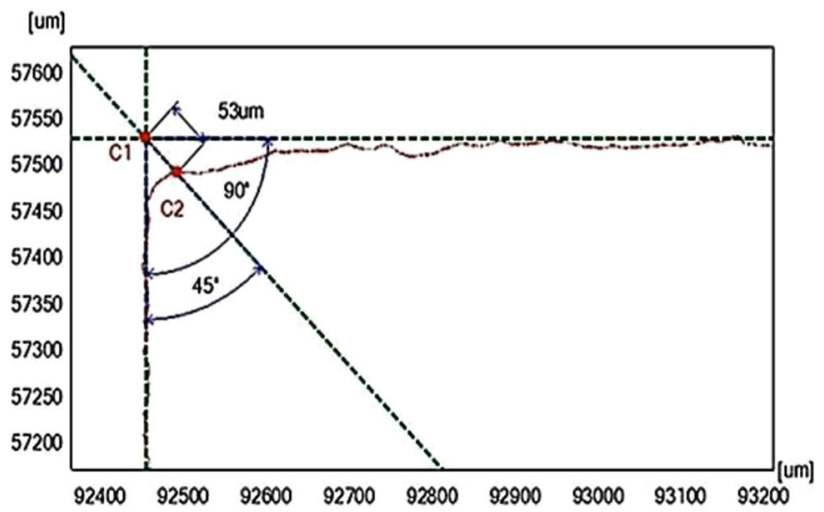

(a) comparatively less energy and thus results in smaller crater size. Hence, with increase in servo sensitivity $\left(\mathrm{S}_{\mathrm{c}}\right)$ there is a reduction in surface roughness $\left(\mathrm{R}_{\mathrm{a}}\right)$ value. At the time due to this decreased inter-electrode gap, the ratio of the gap force shared between the wire electrode and dielectric increases, thus the net force transferred to the wire electrode increases. Due to this increase in net gap force wire deflection as well as corner error $\left(\mathrm{C}_{\mathrm{e}}\right)$ increases with increase in servo sensitivity $\left(\mathrm{S}_{\mathrm{c}}\right)$.

It is finally observed that, all parameters except wire tension $\left(\mathrm{W}_{\mathrm{t}}\right)$, influence machining speed $\left(\mathrm{V}_{\mathrm{c}}\right)$ and corner error $\left(\mathrm{C}_{\mathrm{e}}\right)$ in a similar manner i.e. the parameter that increases the corner error also increases the machining speed. From the present experimental investigation, it is observed that higher value of $\mathrm{W}_{\mathrm{t}}$ is always desirable for achieving a better corner accuracy but at the same time, it may be pointed out that there is an upper limit of this value to avoid wire breakage. A similar trend is observed for surface roughness $\left(R_{\mathrm{a}}\right)$ value in respect of $T_{\text {on }}, A_{\text {on }}, T_{\text {off }}$ and $A_{\text {off. }}$ Thus, it is observed clearly that it is impossible to achieve the best corner accuracy, surface finish along with the best machining speed. Thus, it is evident that to get the desired outcome, appropriate trade-off between all these response parameters is extremely essential.

\section{Corner error and surface characteristics analysis}

Figures $7(\mathrm{a} \& \mathrm{~b})$ and $8(\mathrm{a} \& \mathrm{~b})$ show the corner profiles and CCI images of WEDM machined surface for two different pulse on time $\left(\mathrm{T}_{\mathrm{on}}\right)$ setting. From Figure $7 \mathrm{a}$ and $7 \mathrm{~b}$, it is clear that the corner error $\left(\mathrm{C}_{\mathrm{e}}\right)$ of the profile largely depends on pulse parameter settings, and gradually increases as the pulse on time $\left(\mathrm{T}_{\mathrm{on}}\right)$ increases. Figure $8 \mathrm{a}$ and 8b exhibit the topography of $\mathrm{Al} 7075$ machined surface. Full of scratch and deep craters, high concentration of molten metal (nuggets) and straits have been clearly visualised on the machined surface. The concentration of molten metal, diameter and depth of the craters depend on the energy per pulse i.e. on WEDM pulse parameter setting. Corner error and surface roughness both can be

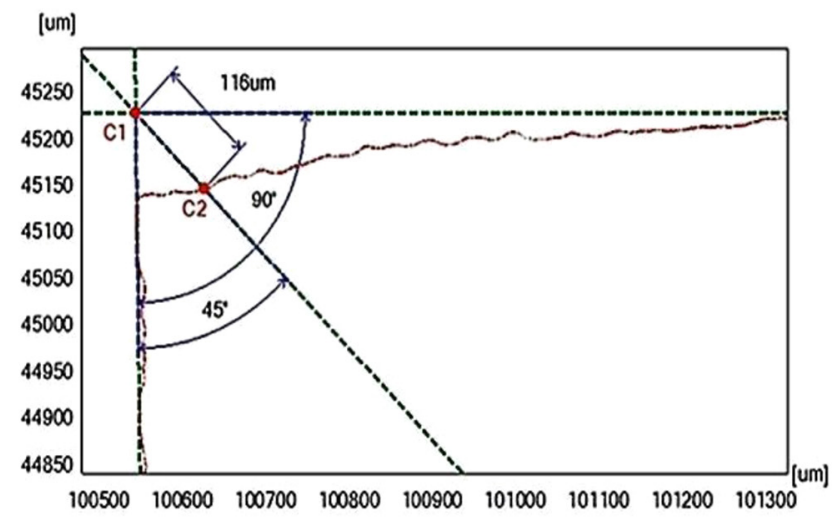

(b)

Fig. 7. Corner profile traced by CV 3200 high precision contour scope (a) $\mathrm{T}_{\text {on }}=0.5 \mu \mathrm{s}(\mathrm{b}) \mathrm{T}_{\mathrm{on}}=0.9 \mu \mathrm{s}$. 


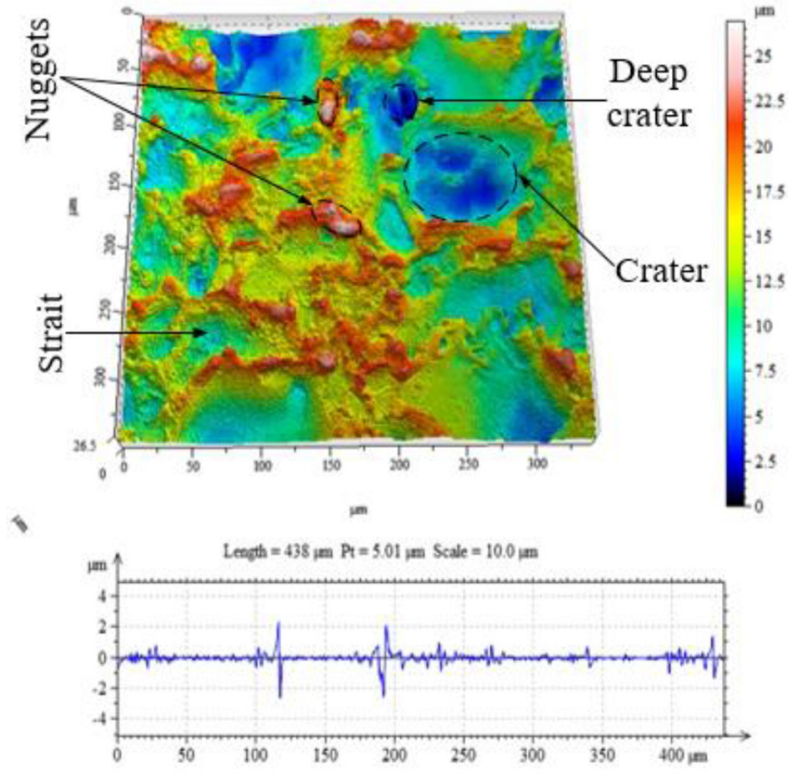

(a)

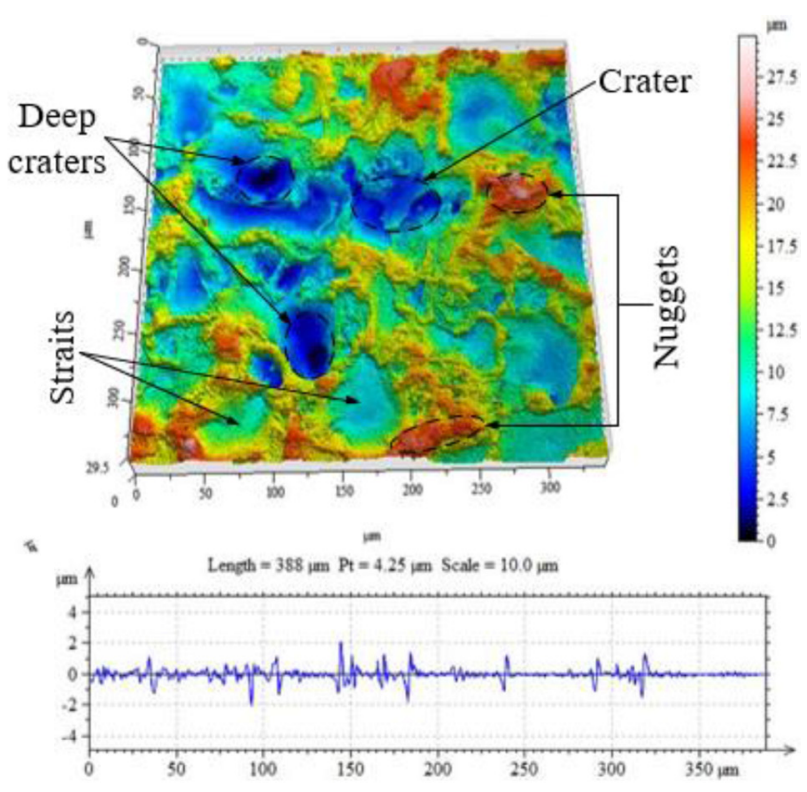

(b)

Fig. 8. High resolution CCI image of the machined surface (a) $\mathrm{T}_{\mathrm{on}}=0.5 \mu \mathrm{s}$ (b) $\mathrm{T}_{\text {on }}=0.9 \mu \mathrm{s}$.

controlled by adjusting pulse parameter setting (i.e. either decreasing pulse on and arc on time or increasing pulse off and arc off time).

\section{Conclusions}

Single pass rough cutting operation in WEDM of Al 7075 has been carried out in this study. The influence of process parameters e.g. pulse on time $\left(\mathrm{T}_{\text {on }}\right)$, arc on time $\left(\mathrm{A}_{\mathrm{on}}\right)$, pulse off time $\left(\mathrm{T}_{\text {off }}\right)$, arc off time $\left(\mathrm{A}_{\text {off }}\right)$, servo voltage $\left(\mathrm{S}_{\mathrm{v}}\right)$, wire tension $\left(W_{t}\right)$ and servo sensitivity $\left(S_{c}\right)$ on machining speed $\left(V_{c}\right)$, corner error $\left(C_{e}\right)$, and surface roughness $\left(R_{a}\right)$ have been investigated. The conclusions of this research work are summarised as follows:

- Pulse on time, pulse off time and arc on time are the main dominating factors on machining speed, corner error and surface roughness in the operating rage of input factors. Wire tension playing a crucial role on corner error. It is observed that corner error significantly decreases with the increase of wire tension. In case of servo sensitivity, machining speed $\left(\mathrm{V}_{\mathrm{c}}\right)$ and corner error $\left(\mathrm{C}_{\mathrm{e}}\right)$ are increases and surface roughness decreases when sensitivity increased.

- The main aim is to find out the best combination of process parameters as known the quality of WEDM, which depends heavily on the input factors. From the experimental investigation it is observed that in order to get the best corner accuracy, the parameters setting should be $\mathrm{T}_{\text {on }}=0.5 \mu \mathrm{s}, \mathrm{T}_{\text {off }}=34 \mu \mathrm{s}, \mathrm{A}_{\text {on }}=0.1 \mu \mathrm{s}, \mathrm{A}_{\text {off }}=$ $30 \mu \mathrm{s}, \quad \mathrm{S}_{\mathrm{v}}=20 \mathrm{~V}, \mathrm{~W}_{\mathrm{t}}=1.6 \mathrm{~kg}, \mathrm{~S}_{\mathrm{c}}=1$. To achieve the maximum possible machining speed, parameters value should be $\mathrm{T}_{\text {on }}=0.9 \mu \mathrm{s}, \mathrm{T}_{\text {off }}=14 \mu \mathrm{s}, \mathrm{A}_{\text {on }}=0.5 \mu \mathrm{s}, \mathrm{A}_{\text {off }}=$ $10 \mu \mathrm{s}, \mathrm{S}_{\mathrm{v}}=10 \mathrm{~V}, \mathrm{~W}_{\mathrm{t}}=1.6 \mathrm{~kg}, \mathrm{~S}_{\mathrm{c}}=5$ and for minimum surface roughness value, parameter setting should be $\mathrm{T}_{\text {on }}=0.5 \mu \mathrm{s}, \mathrm{T}_{\text {off }}=34 \mu \mathrm{s}, \mathrm{A}_{\mathrm{on}}=0.1 \mu \mathrm{s}, \mathrm{A}_{\mathrm{off}}=30 \mu \mathrm{s}, \mathrm{S}_{\mathrm{v}}=$ $10 \mathrm{~V}, \mathrm{~W}_{\mathrm{t}}=0.8 \mathrm{~kg}, \mathrm{~S}_{\mathrm{c}}=5$.

- It is impossible to achieve simultaneously the best corner accuracy and surface finish along with the maximum machining speed. Thus, a systematic trade-off between the response parameters is extremely essential.

- Majority of the input parameters influences the response parameters in a similar manner i.e. the change in input parameters that tends to improve the machining speed also deteriorates the corner accuracy and surface finish.

This research study will be extremely useful for producing high precision job with better surface quality in wire electro-discharge machining of $\mathrm{Al} 7075$ in modern manufacturing industries.

\section{References}

1. A. Gloria, R. Montanari, M. Richetta, V. Varone, Alloys for aeronautic applications: state of the art and perspectives, Metals 9 (2019) 662

2. A. Giridharan, G.L. Samuel, Investigation into erosion rate of AISI 4340 steel during wire electrical discharge turning process, Mach. Sci. Technol. 22 (2018) 187-198

3. S.S. Nain, D. Garg, S. Kumar, Evaluation and analysis of cutting speed, wire wear ratio, and dimensional deviation of wire electric discharge machining of super alloy udimet-L605 using support vector machine and grey relational analysis, Adv. Manuf. 6 (2018) 225-246

4. M.A. Singh, D.K. Sarma, O. Hanzel, J. Sedláček, P. Šajgalík, Surface characteristics and erosion phenomena in WEDM of alumina composites, Mater. Manuf. Processes. 33 (2018) 1815-1821 
5. B.C. Kandpal, J. Kumar, H. Singh, Investigations into electrical discharge machining of fabricated AA 6061/10\% $\mathrm{Al}_{2} \mathrm{O}_{3}$ aluminium-based metal matrix composite using OFAT approach, IJAutoC. 3 (2017) 29-43

6. K. Kumar, K. Abhishek, Influence of process parameters on MRR, kerf width and surface roughness during WEDM on Inconel 718: performance analysis of electrode tool material, Int. J. Ind. Syst. Eng. 30 (2018) 298-315

7. G.Selvakumar, K.G.T. Kuttalingam, M. Selvaraj, J. Manohar, Enhancing die corner accuracy using path modification strategy in wire electrical discharge machining of Monel 400, Proc. IMechE, Part C J. Mech. Eng. Sci. 232 (2018) 207-216

8. R. Bobbili, V. Madhu, A.K. Gogia, Effect of wire-EDM machining parameters on surface roughness and material removal rate of high strength armor steel, Mater. Manuf. Processes. 28 (2013) 364-368

9. S. Sarkar, M. Sekh, S. Mitra, B. Bhattacharyya, A novel method of determination of wire lag for enhanced profile accuracy in WEDM, Precis. Eng. 35 (2011) 339-347
10. H. Abyar, A. Abdullah, A. Akbarzadeh, Analyzing wire deflection errors of WEDM process on small arced corners, J. Manuf. Process. 36 (2018) 216-223

11. S. Lal, S. Kumar, Z.A. Khan, A.N. Siddiquee, Multi-response optimization of wire electrical discharge machining process parameters for $\mathrm{Al} 7075 / \mathrm{Al}_{2} \mathrm{O}_{3} / \mathrm{SiC}$ hybrid composite using taguchi-based grey relational analysis, Proc. IMechE, Part B J. Eng. Manuf. 229 (2015) 229-237

12. T.B. Rao, A.G. Krishna, Selection of optimal process parameters in WEDM while machining Al7075/SiCp metal matrix composites, Int. J. Adv. Manuf. Technol. 73 (2014) 299-314

13. M.H. Gadallah, H.M. Abdu, Modeling and optimization of laser cutting operations, Manuf. Rev. 2 (2015) $1-15$

14. H.A. Hegab, M.H. Gadallah, A.K. Esawi, Modeling and optimization of Electrical Discharge Machining (EDM) using statistical design, Manuf. Rev. 1 (2015) 20-40

Cite this article as: Kingshuk Mandal, Dipankar Bose, Souren Mitra, Soumya Sarkar, Experimental investigation of process parameters in WEDM of Al 7075 alloy, Manufacturing Rev. 7, 30 (2020) 